\title{
Effect of samarium nanoparticles on optical properties of zinc borotellurite glass system
}

\begin{abstract}
The glass series of samarium nanoparticles (NPs) doped zinc borotellurite glasses were successfully fabricated by using conventional melt-quenching technique. The structural properties of the prepared glasses were investigated by X-ray diffraction (XRD) analysis and FTIR analysis. It was confirmed that the prepared glasses are amorphous in nature. The bonding parameters of the glasses were analyzed by using FTIR analysis and were found the formation of non-bridging oxygen. The density of these glasses were measured and found to be increased with increasing samarium NPs content. The optical absorption spectra of these glasses were revealed that the fundamental absorption edge shifts to higher wavelengths as the content of Sm2O3 (NPs) increases. The optical energy band gap are found to be decreased linearly with an increasing samarium NPs concentration which is due to the formation of non-bridging oxygen in the glass system.
\end{abstract}

Keyword: Borotellurite glass; Optical band gap; Samarium nano particles 